\title{
ON THE CONTROLLABILITY OF FRACTIONAL DYNAMICAL SYSTEMS
}

\author{
KRISHNAN BALACHANDRAN, JAYAKUMAR KOKILA \\ Department of Mathematics \\ Bharathiar University, Coimbatore 641 046, India \\ e-mail: \{kb.maths.bu, kokimaths\} @gmail.com
}

\begin{abstract}
This paper is concerned with the controllability of linear and nonlinear fractional dynamical systems in finite dimensional spaces. Sufficient conditions for controllability are obtained using Schauder's fixed point theorem and the controllability Grammian matrix which is defined by the Mittag-Leffler matrix function. Examples are given to illustrate the effectiveness of the theory.
\end{abstract}

Keywords: controllability, fractional differential equations, Mittag-Leffler function.

\section{Introduction}

In the last three decades, interest in fractional calculus has experienced rapid growth and at present we can find many papers devoted its theoretical and application aspects (see the work of Machado et al. (2011) and the references therein). Fractional order models of real systems are often more adequate than the usually used integer order models in electrochemistry (Ichise et al., 1971), advection dispersion models (Benson et al., 2000), anomalous diffusion (Metzler and Klafter, 2000), viscoelastic materials (Renardy et al., 1987), fractal networks (Al Akaidi, 2004; Arena et al., 2000; West et al., 2003) and robotics (Valerio and Sa da Costa, 2004), etc. This is mainly due to the fact that the description of some systems is more accurate when the fractional derivative is used.

For example, consider the time fractional advectiondispersion equation obtained by Liu et al. (2003) by replacing the time-derivative in the advection-dispersion equation by a generalized derivative of order $\alpha$ with $0<$ $\alpha \leq 1$,

$$
\begin{aligned}
\frac{\partial^{\alpha} C(x, t)}{\partial t^{\alpha}} & =\nu \frac{\partial C(x, t)}{\partial x}+D \frac{\partial^{2} C(x, t)}{\partial x^{2}}, \\
C(x, 0) & =C_{0}(x), x \geq 0,
\end{aligned}
$$

where $x>0, t>0, D>0, \nu \geq 0$. From the mathematical point of view, the fractional derivative dates back two centuries, but it was not until much later that substantial theoretical research into fractional derivatives and integrals developed. One can see the monographs of Kilbas et al. (2006), Miller and Ross (1993), Oldham and Spanier
(1974) as well as Podlubny (1999a) for clear exposition of fractional calculus. On the other hand, the theory of fractional differential equations has been extensively discussed in the literature (see the works of Balachandran and Kiruthika (2010), Balachandran et al. (2011), Balachandran and Trujillo (2010), Bonilla et al. (2007) and the references therein).

Qualitative behaviours such as the observability, controllability, stability, stabilizability of fractional dynamical systems are the current issues dealt with by researchers. Mainly, the controllability of dynamical systems is widely used in the analysis and design of control systems. Any control system is said to be controllable if every state corresponding to this process can be affected or controlled in respective time by some control signals. Balachandran and Dauer (1987), Klamka (1993; 2008) as well as Karthikeyan and Balachandran (2011) extensively discussed the controllability results for linear and nonlinear integer order dynamical systems in finite dimensional spaces.

Due to the absence of appropriate mathematical methods, fractional-order dynamical systems have only been studied marginally in the theory and practice of control systems. Some successful attempts have been undertaken, but generally the study in the time domain has almost been avoided. However, during recent years a renewed interest has been devoted to fractional order systems in the area of automatic control. Oustaloup (1991) initiated the first framework for non-integer order systems in the automatic control area. Fractional-order control is the use of fractional calculus in the aforementioned top- 
ics, the system being modeled in a classical way or as a fractional one.

From a certain point of view, the applications of fractional calculus have experienced an evolution analogous to that of control following two parallel paths depending on the starting point: the time domain or the frequency domain. Whilst the applications in dynamic system modeling have used, except in some cases of electrochemistry, the time domain, the applications in control have been developed (Machado, 1997) in the frequency domain. Podlubny (1999b) proposed a generalization of the PID controller, namely, the $P I^{\lambda} D^{\mu}$-controller, involving an integrator of order $\lambda$ and a differentiator of order $\mu$. He also demonstrated a better response of this type of controller, in comparison with the classical PID-controller, when used for the control of fractional-order systems. The integrodifferential equation defining the control action of a fractional order PID controller is given by

$$
u(t)=K_{p} e(t)+K_{i} D^{\lambda} e(t)+K_{d} D^{\mu} e(t) .
$$

Numerous applications have demonstrated that $P I^{\lambda} D^{\mu_{-}}$ controllers perform sufficiently better in the control of fractional-order dynamical systems than the classical PID-controllers.

There are very few contributions regarding the controllability of fractional dynamical systems in the literature. Chen et al. (2006) proposed robust controllability for interval fractional-order linear time invariant systems, whereas Adams and Hartley (2008) studied finite time controllability for fractional systems. Monje et al. (2010) discussed fractional-order systems and controls with fundamental ideas and applications. Shamardan et al. (2000) extended some basic results on the controllability and observability of linear discrete-time fractional-order systems and developed some new concepts inherent to fractionalorder systems with analytical methods for checking their properties.

The analysis of controllability and observability of continuous and discrete time fractional order systems modeled by fractional state space equations was provided by Bettayeb and Djennoune (2008) as well as Guermah et al. (2008), respectively. Klamka (2010) addressed the minimum energy control problem of infinite-dimensional fractional-discrete time linear systems and established necessary and sufficient conditions for exact controllability. Recently, Balachandran et al. (2012) obtained controllability results for nonlinear fractional dynamical systems using Schauder's fixed point theorem.

It should be mentioned that the theory of controllability for nonlinear fractional dynamical systems is still in the development process with solutions far from being satisfactory. Motivated by this fact, the main aim of the present article is to present controllability results for nonlinear fractional dynamical systems with the choice of boundedness of Do (1990) for the nonlinear function. Sufficient conditions for controllability are established using Schauder's fixed point theorem and fractional calculus. The paper is organised as follows. In Section 2, some well known fractional operators, special functions and the solution representation of fractional differential equations are given. Section 3 is devoted to the formulation of linear and nonlinear fractional dynamical systems. Sufficient conditions for controllability results are established using Schauder's fixed point theorem and fractional calculus. Finally, in Section 4, examples are provided to illustrate the effectiveness of the theory.

\section{Preliminaries}

Let $p, q>0$, with $n-1<p<n, n-1<q<n, n \in \mathbb{N}$, $D$ being the usual differential operator. Let $\mathbb{R}^{m}$ be the $m$ dimensional Euclidean space, $\mathbb{R}_{+}=[0, \infty)$, and suppose $f \in L_{1}\left(\mathbb{R}_{+}\right)$. The following definitions and properties are well known, for $p, q>0$ and $f$ as a suitable function (see, e.g., Kilbas et al., 2006; Samko et al., 1993).

(a) Riemann-Liouville fractional operators (left sided):

$$
\begin{aligned}
\left(I_{0+}^{q} f\right)(x) & =\frac{1}{\Gamma(q)} \int_{0}^{x}(x-t)^{q-1} f(t) \mathrm{d} t, \\
\left(D_{0+}^{q} f\right)(x) & =D^{n}\left(I_{0+}^{n-q} f\right)(x) .
\end{aligned}
$$

(b) Caputo fractional derivative (left sided):

$$
\left({ }^{C} D_{0+}^{q} f\right)(x)=\left(I_{0+}^{n-q} D^{n} f\right)(x) .
$$

In particular, $I_{0+}^{q}{ }^{C} D_{0+}^{q} f(t)=f(t)-f(0)$, where $0<$ $q<1$.

The following is a well-known relation, for a finite interval $[a, b] \in \mathbb{R}_{+}$:

$$
\begin{array}{r}
\left(D_{a+}^{q} f\right)(x)=\left({ }^{C} D_{a+}^{q} f\right)(x)+\sum_{j=0}^{n-1} \frac{f^{(j)}(a)(x-a)^{j-q}}{\Gamma(1+j-q)}, \\
\text { for } n=\Re(q)+1 .
\end{array}
$$

The Laplace transform of the Caputo fractional derivative is given as

$$
\mathcal{L}\left\{{ }^{C} D_{0+}^{q} f(t)\right\}(s)=s^{q} F(s)-\sum_{k=0}^{n-1} f^{(k)}\left(0^{+}\right) s^{q-1-k} .
$$

Riemann-Liouville fractional derivatives have singularity at zero (Chikrii and Matichin, 2010) and fractional differential equations in the Riemann-Liouville sense require initial conditions of special form lacking physical interpretation. To overcome this difficulty, Caputo (1967) introduced a new definition of the fractional derivative but, in general, neither the Riemann-Liouville nor Caputo fractional operators possess semigroup or commutative properties, which are inherent to the derivatives of 
integer orders. Therefore, the concept of sequential fractional differential equations is discussed by Kilbas et al. (2006) as well as Miller and Ross (1993).

(c) Linear sequential derivative: For $n \in \mathbb{N}$, the sequential fractional derivative for a suitable function $y(t)$ is defined by

$$
y^{(k q}:=\left(\mathbf{D}^{k q} y\right)(t)=\left(\mathbf{D}^{q} \mathbf{D}^{(k-1) q} y\right)(t),
$$

where $k=1, \ldots, n,\left(\mathbf{D}^{0} y\right)(t)=y(t)$, and $\mathbf{D}^{q}$ is any fractional differential operator (here we refer to it as ${ }^{C} D_{0+}^{q}$ ).

For brevity, let us take $I_{0+}^{q}$ as $I^{q}$ and ${ }^{C} D_{0+}^{q}$ as ${ }^{C} D^{q}$, and the fractional derivative is taken in the Caputo sense.

(d) Mittag-Leffler function:

$$
E_{q, p}(z)=\sum_{k=0}^{\infty} \frac{z^{k}}{\Gamma(k q+p)} \text { for } q, p>0 .
$$

The general Mittag-Leffler function satisfies

$$
\int_{0}^{\infty} e^{-t} t^{p-1} E_{q, p}\left(t^{q} z\right) \mathrm{d} t=\frac{1}{1-z} \text { for }|z|<1 .
$$

The Laplace transform of $E_{q, p}(z)$ follows from the integral

$$
\int_{0}^{\infty} e^{-s t} t^{p-1} E_{q, p}\left( \pm a t^{q}\right) \mathrm{d} t=\frac{s^{q-p}}{\left(s^{q} \mp a\right)} .
$$

That is,

$$
\mathcal{L}\left\{t^{p-1} E_{q, p}\left( \pm a t^{q}\right)\right\}(s)=\frac{s^{q-p}}{\left(s^{q} \mp a\right)},
$$

for $\Re(s)>|a|^{\frac{1}{q}}$ and $\Re(p)>0$. In particular, for $p=1$,

$$
E_{q, 1}\left(\lambda z^{q}\right)=E_{q}\left(\lambda z^{q}\right)=\sum_{k=0}^{\infty} \frac{\lambda^{k} z^{k q}}{\Gamma(q k+1)}, \quad \lambda, z \in \mathbb{C}
$$

have the interesting property

$$
{ }^{C} D^{q} E_{q}\left(\lambda t^{q}\right)=\lambda E_{q}\left(\lambda t^{q}\right)
$$

and

$$
\mathcal{L}\left\{E_{q}\left( \pm a t^{q}\right)\right\}(s)=\frac{s^{q-1}}{\left(s^{q} \mp a\right)} \quad \text { for } p=1 .
$$

(e) Solution representation: Consider the linear fractional differential equation of the form

$$
\begin{aligned}
{ }^{C} D^{q} x(t) & =A x(t)+f(t), \quad t \in[0, T], \\
x(0) & =x_{0},
\end{aligned}
$$

where $0<q<1, x \in \mathbb{R}^{n}$, and $A$ is an $n \times n$ matrix. In order to find a solution, apply the Laplace transform to both the sides and use the Laplace transform of the Caputo derivative to get

$$
s^{q} X(s)-s^{q-1} x(0)=A X(s)+F(s) .
$$

Applying the inverse Laplace transform to both the sides, we have

$$
\begin{aligned}
\mathcal{L}^{-1}\{X(s)\}= & \mathcal{L}^{-1}\left\{s^{q-1}\left(s^{q} I-A\right)^{-1}\right\} x_{0} \\
& +\mathcal{L}^{-1}\{F(s)\} * \mathcal{L}^{-1}\left\{\left(s^{q} I-A\right)^{-1}\right\} .
\end{aligned}
$$

Finally, substituting the Laplace transformation of the Mittag-Leffler function, we get the solution to the given equation (Chikrii and Matichin, 2008; Kexue and Jigen, 2011),

$$
\begin{aligned}
x(t)= & E_{q}\left(A t^{q}\right) x_{0} \\
& +\int_{0}^{t}(t-s)^{q-1} E_{q, q}\left(A(t-s)^{q}\right) f(s) \mathrm{d} s .
\end{aligned}
$$

where $E_{q}\left(A t^{q}\right)$ is the matrix extension of the abovementioned Mittag-Leffler function with the following representation:

$$
E_{q}\left(A t^{q}\right)=\sum_{k=0}^{\infty} \frac{A^{k} t^{k q}}{\Gamma(k q+1)}
$$

with the property ${ }^{C} D^{q} E_{q}\left(A t^{q}\right)=A E_{q}\left(A t^{q}\right)$.

\section{Controllability results}

Consider a linear dynamical system represented by the fractional differential equation of the form

$$
\begin{aligned}
{ }^{C} D^{q} x(t) & =A x(t)+B u(t), \quad t \in[0, b]:=J, \\
x(0) & =x_{0},
\end{aligned}
$$

where $0<q<1, x \in \mathbb{R}^{n}, u \in \mathbb{R}^{m}$, and $A$ is an $n \times n$ matrix while $B$ is an $n \times m$ matrix. Then the solution of the system (1) is given by (Chikrii and Matichin, 2008)

$$
\begin{aligned}
x(t)= & E_{q}\left(A t^{q}\right) x_{0} \\
& +\int_{0}^{t}(t-s)^{q-1} E_{q, q}\left(A(t-s)^{q}\right) B u(s) \mathrm{d} s .(2)
\end{aligned}
$$

In particular, the solution (2) satisfies the fractional differential equation (1). Similarly to the conventional controllability concept, the controllability of the fractional dynamical system is defined as follows (Chen et al., 2006).

Definition 1. The system (1) is said to be controllable on $J$ if for every $x_{0}, x_{1} \in \mathbb{R}^{n}$ there exists a control $u(t)$ such that the solution $x(t)$ of Eqn. (1) satisfies the conditions $x(0)=x_{0}$ and $x(b)=x_{1}$. 
Theorem 1. The linear control system (1) is controllable on $[0, b]$ if and only if the controllability Grammian matrix

$$
\begin{array}{r}
W=\int_{0}^{b}(b-s)^{q-1}\left[E_{q, q}\left(A(b-s)^{q}\right) B\right] \\
{\left[E_{q, q}\left(A(b-s)^{q}\right) B\right]^{*} \mathrm{~d} s}
\end{array}
$$

is positive definite for some $b>0$.

Proof. Since $W$ is positive definite, it is non-singular and therefore its inverse is well defined. Take the control function

$$
u(t)=B^{*} E_{q, q}\left(A^{*}(b-t)^{q}\right) W^{-1}\left[x_{1}-E_{q}\left(A b^{q}\right) x_{0}\right] .
$$

Here $*$ indicates the matrix transpose and the norm of a matrix is taken as the usual matrix norm. Further, observe that the control (3) steers the system (1) from $x_{0}$ to $x_{1}$.

Substituting $t=b$ in (2) and inserting (3), we get

$$
\begin{aligned}
x(b)= & E_{q}\left(A b^{q}\right) x_{0} \\
& +\int_{0}^{b}(b-s)^{q-1} E_{q, q}\left(A(b-s)^{q}\right) B B^{*} \\
& \times E_{q, q}\left(A^{*}(b-s)^{q}\right) W^{-1}\left[x_{1}-E_{q}\left(A b^{q}\right) x_{0}\right] \mathrm{d} s \\
= & E_{q}\left(A b^{q}\right) x_{0}+W W^{-1}\left[x_{1}-E_{q}\left(A b^{q}\right) x_{0}\right] \\
x(b)= & x_{1} .
\end{aligned}
$$

Thus (1) is controllable.

On the other hand, if it is not positive definite, there exists a nonzero $y$ such that

$$
y^{*} W y=0,
$$

that is,

$$
\begin{aligned}
y^{*} \int_{0}^{b}(b-s)^{q-1} E_{q, q}\left(A(b-s)^{q}\right) B & \\
\times B^{*} E_{q, q}\left(A^{*}(b-s)^{q}\right) y \mathrm{~d} s & =0, \\
y^{*} E_{q, q}\left(A(b-s)^{q}\right) B & =0 \text { on }[0, b] .
\end{aligned}
$$

Let $x_{0}=\left[E_{q}\left(A b^{q}\right)\right]^{-1} y$. By assumption, there exists an input $u$ such that it steers $x_{0}$ to the origin in the interval $[0, b]$. It follows that

$$
\begin{aligned}
x(b)=0= & E_{q}\left(A b^{q}\right) x_{0} \\
& +\int_{0}^{b}(b-s)^{q-1} E_{q, q}\left(A(b-s)^{q}\right) B u(s) \mathrm{d} s \\
0= & y+\int_{0}^{b}(b-s)^{q-1} E_{q, q}\left(A(b-s)^{q}\right) B u(s) \mathrm{d} s .
\end{aligned}
$$

Then

$0=y^{*} y+\int_{0}^{b}(b-s)^{q-1} y^{*} E_{q, q}\left(A(b-s)^{q}\right) B u(s) \mathrm{d} s$.

But the second term is zero, leading to the conclusion that $y^{*} y=0$. This is a contradiction to $y \neq 0$. Thus $W$ is positive definite. Hence the proof is completed.
Consider a nonlinear fractional dynamical system represented by the fractional differential equation of the form

$$
\begin{aligned}
{ }^{C} D^{q} x(t) & =A x(t)+B u(t)+f(t, x(t), u(t)), t \in J \\
x(0) & =x_{0}
\end{aligned}
$$

where $0<q<1, x \in \mathbb{R}^{n}, u \in \mathbb{R}^{m}$, and $A, B$ are matrices of dimensions $n \times n$ and $n \times m$, respectively, while $f: J \times \mathbb{R}^{n} \times \mathbb{R}^{m} \rightarrow \mathbb{R}^{n}$ is a given function.

Let us introduce the following notation. Denote by $\mathcal{Q}$ the Banach space of continuous $\mathbb{R}^{n} \times \mathbb{R}^{m}$ valued functions defined on the interval $J$ with the norm $\|(x, u)\|=$ $\|x\|+\|u\|$, where $\|x\|=\sup \{|x(t)|: t \in J\}$ and $\|u\|=$ $\sup \{|u(t)|: t \in J\}$. That is, $\mathcal{Q}=C_{n}(J) \times C_{m}(J)$, where $C_{n}(J)$ is the Banach space of continuous $\mathbb{R}^{n}$ valued functions defined on the interval $J$ with the sup norm. For each $(z, v) \in \mathcal{Q}$, consider the fractional dynamical system

$$
\begin{aligned}
{ }^{C} D^{q} x(t) & =A x(t)+B u(t)+f(t, z(t), v(t)), \\
x(0) & =x_{0}
\end{aligned}
$$

Then the solution is given by

$$
\begin{aligned}
& x(t) \\
& =E_{q}\left(A t^{q}\right) x_{0} \\
& \quad+\int_{0}^{t}(t-s)^{q-1} E_{q, q}\left(A(t-s)^{q}\right) B u(s) \mathrm{d} s \\
& \quad+\int_{0}^{t}(t-s)^{q-1} E_{q, q}\left(A(t-s)^{q}\right) f(s, z(s), v(s)) \mathrm{d} s .
\end{aligned}
$$

For our convenience, let us introduce the following notation:

$$
\begin{aligned}
a_{1} & =\sup \left\|E_{q, q}\left(A(b-t)^{q}\right)\right\|, \\
a_{2} & =\sup \left\|E_{q}\left(A t^{q}\right) x_{0}\right\|, \\
\gamma_{i} & =4 a_{1}^{2} b^{q}\left\|\alpha_{i}\right\|\left\|B^{*}\right\|\left\|W^{-1}\right\| q^{-1}, \\
\beta_{i} & =4 a_{1} b^{q}\left\|\alpha_{i}\right\| q^{-1} \\
d_{1} & =4 a_{1}\left\|B^{*}\right\|\left\|W^{-1}\right\|\left[\left|x_{1}\right|+a_{2}\right], \\
d_{2} & =4 a_{2}, \\
a & =\max \left\{a_{1} b^{q}\|B\| q^{-1}, 1\right\}, \\
c_{i} & =\max \left\{\gamma_{i}, \beta_{i}\right\}, \\
d & =\max \left\{d_{1}, d_{2}\right\} .
\end{aligned}
$$

Now we prove the main result of the paper.

Theorem 2. Let the function $f$ satisfy the condition

$$
\|f(t, x, u)\| \leq \sum_{i=1}^{p} \alpha_{i}(t) \phi_{i}(x, u)
$$


where $\phi_{i}: \mathbb{R}^{n} \times \mathbb{R}^{m} \rightarrow \mathbb{R}_{+}$are measurable functions and $\alpha_{i}: J \rightarrow \mathbb{R}_{+}$are $L_{1}$ functions for $i=1,2, \ldots, p$. Suppose that the linear system (1) is controllable, and if the following equality holds:

$$
\begin{aligned}
& \limsup _{r \rightarrow \infty}\left(r-\sum_{i=1}^{p} c_{i} \sup \left\{\phi_{i}(x, u)\right.\right. \\
&:\|(x, u)\| \leq r\})=+\infty
\end{aligned}
$$

Then the nonlinear system (4) is controllable on J.

Proof. Define the operator $\mathcal{P}: \mathcal{Q} \rightarrow \mathcal{Q}$ by

$$
\mathcal{P}(z, v)=(x, u)
$$

where

$$
\begin{aligned}
u(t)= & B^{*} E_{q, q}\left(A^{*}(b-t)^{q}\right) W^{-1}\left[x_{1}-E_{q}\left(A b^{q}\right) x_{0}\right. \\
& \left.-\int_{0}^{b}(b-s)^{q-1} E_{q, q}\left(A(b-s)^{q}\right) f(s, z, v) \mathrm{d} s\right]
\end{aligned}
$$

and

$$
\begin{aligned}
x(t)= & E_{q}\left(A t^{q}\right) x_{0} \\
& +\int_{0}^{t}(t-s)^{q-1} E_{q, q}\left(A(t-s)^{q}\right) B u(s) \mathrm{d} s \\
& +\int_{0}^{t}(t-s)^{q-1} E_{q, q}\left(A(t-s)^{q}\right) f(s, z, v) \mathrm{d} s .
\end{aligned}
$$

Now let

$$
\psi_{i}(r)=\sup \left\{\phi_{i}(x, u):\|(x, u)\| \leq r\right\} .
$$

Since (6) holds, there exists $r_{0}>0$ such that

$$
r_{0}-\sum_{i=1}^{p} c_{i} \psi_{i}\left(r_{0}\right) \geq d
$$

which implies

$$
\sum_{i=1}^{p} c_{i} \psi_{i}\left(r_{0}\right)+d \leq r_{0}
$$

Then, by (8) and (9),

$$
\begin{aligned}
& \|u(t)\| \\
& \leq a_{1}\left\|B^{*}\right\|\left\|W^{-1}\right\|\left[\left|x_{1}\right|+a_{2}+b^{q} a_{1} q^{-1} \sum_{i=1}^{p}\left\|\alpha_{i}\right\| \psi_{i}\left(r_{0}\right)\right] \\
& \leq\left[\frac{d_{1}}{4 a}+\frac{1}{4 a} \sum_{i=1}^{p} \gamma_{i} \psi_{i}\left(r_{0}\right)\right] \leq \frac{1}{4 a}\left[d+\sum_{i=1}^{p} c_{i} \psi_{i}\left(r_{0}\right)\right] .
\end{aligned}
$$

$$
\begin{aligned}
& \|x(t)\| \\
& \leq a_{2}+\frac{a_{1} b^{q}\|B\|}{4 a q}\left[d+\sum_{i=1}^{p} c_{i} \psi_{i}\left(r_{0}\right)\right] \\
& \quad+\frac{a_{1} b^{q}}{q} \sum_{i=1}^{p}\left\|\alpha_{i}\right\| \psi_{i}\left(r_{0}\right) \\
& \leq \frac{d}{4}+\frac{1}{4}\left[d+\sum_{i=1}^{p} c_{i} \psi_{i}\left(r_{0}\right)\right]+\frac{1}{4} \sum_{i=1}^{p} c_{i} \psi_{i}\left(r_{0}\right) \\
& \leq \frac{1}{2}\left[d+\sum_{i=1}^{p} c_{i} \psi_{i}\left(r_{0}\right)\right] .
\end{aligned}
$$

Therefore, $|u(s)| \leq r_{0} / 4 a$, for all $s \in J$, and hence $\|u\| \leq r_{0} / 4 a$, which gives $\|x\| \leq r_{0} / 2$. Thus, we have proved that, if

$$
\mathcal{Q}\left(r_{0}\right)=\left\{(z, v) \in \mathcal{Q}:\|z\| \leq r_{0} \text { and }\|v\| \leq r_{0}\right\},
$$

then $\mathcal{P}$ maps $\mathcal{Q}\left(r_{0}\right)$ into itself. Now let us take $t_{1}, t_{2} \in J$ with $t_{1}<t_{2}$, and for all $(x, u) \in \mathcal{Q}(r)$ we have to show that $\mathcal{P}[\mathcal{Q}(r)]$ is equicontinuous for all $r>0$ :

$$
\begin{aligned}
& \left\|u\left(t_{1}\right)-u\left(t_{2}\right)\right\| \\
& =\|\left[B ^ { * } E _ { q , q } \left(A^{*}\left(b-t_{1}\right)^{q}\right.\right. \\
& \left.\quad-B^{*} E_{q, q}\left(A^{*}\left(b-t_{2}\right)^{q}\right)\right] W^{-1}\left[x_{1}+E_{q}\left(A b^{q}\right) x_{0}\right. \\
& \quad+\int_{0}^{b}(b-s)^{q-1} E_{q, q}\left(A^{*}(b-s)^{q} f(s, x(s), u(s)) \mathrm{d} s\right] \| \\
& \leq \| B^{*} E_{q}\left(A^{*}\left(b-t_{1}\right)^{q}\right. \\
& \quad-B^{*} E_{q}\left(A^{*}\left(b-t_{2}\right)^{q}\right)\|\| W^{-1} \|\left[\left\|x_{1}\right\|+\left\|E_{q}\left(A b^{q}\right) x_{0}\right\|\right. \\
& \quad+\int_{0}^{b}(b-s)^{q-1} \| E_{q, q}\left(A^{*}(b-s)^{q}\left\|\sum_{i=1}^{p}\right\| \alpha_{i} \| \psi_{i}(r) \mathrm{d} s\right] .
\end{aligned}
$$

We have

$$
\begin{aligned}
\| x\left(t_{1}\right) & -x\left(t_{2}\right) \| \\
=\| & E_{q}\left(A t_{1}^{q}\right) x_{0}-E_{q}\left(A t_{2}^{q}\right) x_{0} \\
& +\int_{0}^{t_{1}}\left(t_{1}-s\right)^{q-1} E_{q, q}\left(A\left(t_{1}-s\right)^{q}\right) B u(s) \mathrm{d} s \\
& -\int_{0}^{t_{2}}\left(t_{2}-s\right)^{q-1} E_{q, q}\left(A\left(t_{2}-s\right)^{q}\right) B u(s) \mathrm{d} s \\
& +\int_{0}^{t_{1}}\left(t_{1}-s\right)^{q-1} E_{q, q}\left(A\left(t_{1}-s\right)^{q}\right) f \mathrm{~d} s \\
& -\int_{0}^{t_{2}}\left(t_{2}-s\right)^{q-1} E_{q, q}\left(A\left(t_{2}-s\right)^{q}\right) f \mathrm{~d} s \|
\end{aligned}
$$




$$
\begin{aligned}
\leq & \left\|E_{q}\left(A t_{1}^{q}\right) x_{0}-E_{q}\left(A t_{2}^{q}\right) x_{0}\right\| \\
+ & \int_{t_{1}}^{t_{2}}\left(t_{2}-s\right)^{q-1}\left\|E_{q, q}\left(A\left(t_{2}-s\right)^{q}\right)\right\|\|B\|\|u(s)\| \mathrm{d} s \\
+ & \int_{0}^{t_{1}}\left[\left(t_{1}-s\right)^{q-1} \| E_{q, q} A\left(t_{1}-s\right)^{q}\right) \| \\
& \left.-\left(t_{2}-s\right)^{q-1}\left\|E_{q, q}\left(A\left(t_{2}-s\right)^{q}\right)\right\|\right]\|B\|\|u(s)\| \mathrm{d} s \\
+ & \int_{0}^{t_{1}}\left[\left(t_{1}-s\right)^{q-1}\left\|E_{q, q}\left(A\left(t_{1}-s\right)^{q}\right)\right\|\right. \\
& \left.-\left(t_{2}-s\right)^{q-1}\left\|E_{q, q}\left(A\left(t_{2}-s\right)^{q}\right)\right\|\right]\|f\| \mathrm{d} s \\
+ & \int_{t_{1}}^{t_{2}}\left(t_{2}-s\right)^{q-1}\left\|E_{q, q}\left(A\left(t_{2}-s\right)^{q}\right)\right\|\|f\| \mathrm{d} s \\
\leq & \left\|E_{q}\left(A t_{1}^{q}\right) x_{0}-E_{q}\left(A t_{2}^{q}\right) x_{0}\right\| \\
& +\int_{t_{1}}^{t_{2}}\left(t_{2}-s\right)^{q-1}\left\|E_{q, q}\left(A\left(t_{2}-s\right)^{q}\right)\right\|\|B\|\|u(s)\| \mathrm{d} s \\
& +\int_{0}^{t_{1}}\left[\left(t_{1}-s\right)^{q-1}\left\|E_{q, q}\left(A\left(t_{1}-s\right)^{q}\right)\right\|\right. \\
& +\int_{t_{1}}^{p}\left(t_{2}-s\right)^{q-1}\left\|E_{q, q}\left(A\left(t_{2}-s\right)^{q}\right)\right\| \\
& \left.+\left(t_{2}-s\right)^{q-1}\left\|E_{q, q}\left(A\left(t_{2}-s\right)^{q}\right)\right\|\right]\|B\|\|u(s)\| \mathrm{d} s \\
& +\int_{0}^{t_{1}} \sum_{i=1}^{p}\left\|\alpha_{i}\right\| \psi_{i}(r)\left[\left\|E_{q, q}\left(A\left(t_{1}-s\right)^{q}\right)\right\|\right. \\
& \times(11)
\end{aligned}
$$

Moreover, for all $(x, u) \in \mathcal{Q}(r)$,

$$
\begin{aligned}
\|u(t)\| \leq & \left\|B^{*}\right\|\left\|E_{q, q}\left(A^{*}(b-t)^{q}\right)\right\|\left\|W^{-1}\right\| \\
& {\left[\left\|x_{1}\right\|+\left\|E_{q}\left(A b^{q}\right) x_{0}\right\|\right.} \\
& +\int_{0}^{b}(b-s)^{q-1} \| E_{q, q}\left(A^{*}(b-s)^{q} \|\right. \\
& \left.\sum_{i=1}^{p}\left\|\alpha_{i}\right\| \psi_{i}(r) \mathrm{d} s\right] .
\end{aligned}
$$

Thus the right-hand side of Eqns. (10) and (11) is independent of $(x, u) \in \mathcal{Q}(r)$ and tends to zero as $t_{1} \rightarrow t_{2}$. Hence $\mathcal{P}[\mathcal{Q}(r)]$ is equicontinuous for all $r>0$ and, by the regularity assumption on $f$, the operator is continuous, and hence it is completely continuous by the application of the Arzela-Ascoli theorem. Since $\mathcal{Q}\left(r_{0}\right)$ is closed, bounded and convex, the Schauder fixed point theorem guarantees that $\mathcal{P}$ has a fixed point $(z, v) \in \mathcal{Q}(r)$ such that $\mathcal{P}(z, v)=(z, v) \equiv(x, u)$. Hence we have

$$
\begin{aligned}
& x(t) \\
& =E_{q}\left(A t^{q}\right) x_{0} \\
& \quad+\int_{0}^{t}(t-s)^{q-1} E_{q, q}\left(A(t-s)^{q}\right) B u(s) \mathrm{d} s \\
& \quad+\int_{0}^{t}(t-s)^{q-1} E_{q, q}\left(A(t-s)^{q}\right) f(s, x(s), u(s)) \mathrm{d} s .
\end{aligned}
$$

Thus, $x(t)$ is the solution of the system (4), and it is easy to verify that $x(b)=x_{1}$. Hence the system (4) is controllable on $J$.

\section{Examples}

In this section we apply the results obtained in the previous section to linear and nonlinear fractional dynamical systems.

Example 1. Consider the following nonlinear fractional dynamical system represented by the scalar fractional differential equation:

$$
\begin{aligned}
{ }^{C} D^{1 / 2} x(t) & =x(t)+u(t)+\sin x(t) \cos u(t), \quad t \in[0,1], \\
x(0) & =x_{0},
\end{aligned}
$$

where $A=B=1, q=1 / 2, b=1, f(t, x(t), u(t))=$ $\sin x(t) \cos u(t)$. The two parameter Mittag-Leffler function is given by

$$
E_{1 / 2,1 / 2}\left((t-s)^{1 / 2}\right)=\sum_{k=0}^{\infty} \frac{(t-s)^{k / 2}}{\Gamma((k+1) / 2)} .
$$

By simple calculation, one can see that the controllability Grammian is

$$
\begin{aligned}
W= & \int_{0}^{1}(1-s)^{-1 / 2}\left[E_{1 / 2,1 / 2}\left((1-s)^{1 / 2}\right)\right] \\
& \times\left[E_{1 / 2,1 / 2}\left((1-s)^{1 / 2}\right)\right]^{*} \mathrm{~d} s \\
= & \int_{0}^{1}(1-s)^{-1 / 2} \sum_{k=0}^{\infty} \frac{(1-s)^{k / 2}}{\Gamma((k+1) / 2)} \\
& \times \sum_{m=0}^{\infty} \frac{(1-s)^{m / 2}}{\Gamma((m+1) / 2)} \mathrm{d} s \\
= & \int_{0}^{1} \sum_{k=0}^{\infty} \sum_{m=0}^{\infty} \frac{(1-s)^{(k+m-1) / 2}}{\Gamma((k+1) / 2) \Gamma((m+1) / 2)} \mathrm{d} s \\
= & \sum_{k=0}^{\infty} \sum_{m=0}^{\infty} \frac{2}{(k+m+1) \Gamma((k+1) / 2) \Gamma((m+1) / 2)} \\
> & 0
\end{aligned}
$$


and the control function is

$$
\begin{aligned}
u(t)= & \sum_{k=0}^{\infty} \frac{(1-t)^{k / 2}}{\Gamma((k+1) / 2)} n_{1}^{-1}\left[x_{1}-\sum_{k=0}^{\infty} \frac{(1)^{k / 2}}{\Gamma((k / 2+1)} x_{0}\right. \\
& \left.-\sum_{k=0}^{\infty} \int_{0}^{1} \frac{(1-s)^{(k-1) / 2}}{\Gamma((k+1) / 2)} \sin x(s) \cos u(s) \mathrm{d} s\right],
\end{aligned}
$$

where

$$
=\sum_{k=0}^{\infty} \sum_{m=0}^{\infty} \frac{2}{(k+m+1) \Gamma((k+1) / 2) \Gamma((m+1) / 2)}
$$

Since $W>0$, the linear system is controllable and the nonlinear function $f(t, x, u)=\sin x \cos u$ does not satisfy the condition stated in Theorem 2. However, by Theorem 3.1 of Balachandran et al. (2012), the nonlinear system (13) is controllable on $[0,1]$.

Example 2. Consider the fractional harmonic oscillator equation (Herrmann, 2011)

$$
\left(m^{C} D^{2 q}+k\right) x(t)=0
$$

where $k$ and $m$ are appropriate constants. Introducing a control variable and a nonlinear forcing term, we get the following controlled fractional harmonic oscillator equation:

${ }^{C} D^{2 q} x(t)+x(t)=u(t)+\frac{x(t)}{1+x^{2}(t)+u^{2}(t)}, \quad t \in J$.

Let us introduce the auxiliary variables $x_{1}(t)=x(t)$ and $x_{2}(t)={ }^{C} D^{q} x_{1}(t)$. Then

$$
\begin{aligned}
{ }^{C} D^{q} x_{1}(t) & ={ }^{C} D^{q} x(t)=x_{2}(t), \\
{ }^{C} D^{q} x_{2}(t) & ={ }^{C} D^{2 q} x(t), \\
& =-x_{1}(t)+u(t)+\frac{x_{1}}{1+x_{1}^{2}(t)+u^{2}(t)} .
\end{aligned}
$$

Hence the above equation has the matrix form

$$
{ }^{C} D^{q} x(t)=A x(t)+B u(t)+f(t, x(t), u(t)),
$$

with

$A=\left(\begin{array}{cc}0 & 1 \\ -1 & 0\end{array}\right), \quad B=\left(\begin{array}{l}0 \\ 1\end{array}\right), \quad x(t)=\left(\begin{array}{l}x_{1}(t) \\ x_{2}(t)\end{array}\right)$

and

$$
f(t, x(t), u(t))=\left(\begin{array}{c}
0 \\
x_{1}(t) \\
\frac{1+x_{1}^{2}(t)+u^{2}(t)}{1+}
\end{array}\right) .
$$

The two parameter Mittag-Leffler matrix function is given by

$$
\begin{aligned}
E_{q, q}\left(A(t-s)^{q}\right) & =\left(\begin{array}{cc}
N_{1}(t-s) & N_{2}(t-s) \\
N_{3}(t-s) & N_{4}(t-s)
\end{array}\right), \\
N_{1}(t-s) & =\sum_{k=0}^{\infty} \frac{(-1)^{k}(t-s)^{2 k q}}{\Gamma(2 k q+q)}, \\
N_{2}(t-s) & =\sum_{k=0}^{\infty} \frac{(-1)^{k}(t-s)^{(2 k+1) q}}{\Gamma((2 k+1) q+q)} \\
N_{3}(t-s) & =\sum_{k=0}^{\infty} \frac{(-1)^{k+1}(t-s)^{(2 k+1) q}}{\Gamma((2 k+1) q+q)}, \\
N_{4}(t-s) & =\sum_{k=0}^{\infty} \frac{(-1)^{k}(t-s)^{2 k q}}{\Gamma(2 k q+q)} .
\end{aligned}
$$

By simple matrix calculation one can see that the controllability matrix

$$
\begin{aligned}
W= & \int_{0}^{b}(b-s)^{q-1}\left[E_{q, q}\left(A(b-s)^{q}\right) B\right] \\
& \times\left[E_{q, q}\left(A(b-s)^{q}\right) B\right]^{*} \mathrm{~d} s \\
= & \int_{0}^{b}(b-s)^{q-1}\left(\begin{array}{cc}
M_{1} & M_{2} \\
M_{3} & M_{4}
\end{array}\right) \mathrm{d} s,
\end{aligned}
$$

where

$$
\begin{aligned}
M_{1} & =N_{2}^{2}(b-s) \\
& =\sum_{k=0}^{\infty} \sum_{m=0}^{\infty} \frac{(-1)^{k+m}(b-s)^{2 q(k+m+1)}}{\Gamma(2 q(k+1)) \Gamma(2 q(m+1))}, \\
M_{2} & =M_{3}=N_{2}(b-s) N_{4}(b-s) \\
& =\sum_{k=0}^{\infty} \sum_{m=0}^{\infty} \frac{(-1)^{k+m}(b-s)^{2 q(k+m)+q)}}{\Gamma(2 q(k+1)) \Gamma(q(2 m+1))}, \\
M_{4} & =N_{4}^{2}(b-s) \\
& =\sum_{k=0}^{\infty} \sum_{m=0}^{\infty} \frac{(-1)^{k+m}(b-s)^{2 q(k+m)}}{\Gamma(q(2 k+1)) \Gamma(q(2 m+1))},
\end{aligned}
$$

is positive definite for any $b>0$. Further, the nonlinear function $f(t, x(t), u(t))$ satisfies the hypothesis of Theorem 2. Observe that the control defined by

$$
\begin{aligned}
u(t)= & B^{*} E_{q, q}\left(A^{*}(b-t)^{q}\right) W^{-1}\left[x_{1}-E_{q}\left(A b^{q}\right) x_{0}\right. \\
& \left.-\int_{0}^{b}(b-s)^{q-1} E_{q, q}\left(A(b-s)^{q}\right) f(s, x, u) \mathrm{d} s\right]
\end{aligned}
$$

steers the system (15) from $x_{0}$ to $x_{1}$, and so the fractional system (15) is controllable on $[0, b]$.

\section{Conclusion}

This paper deals with the controllability of linear and nonlinear fractional dynamical systems. It should be men- 
tioned that the boundedness of the nonlinearity (Do, 1990) assumed here is different from that of Balachandran et al. (2012). Necessary and sufficient conditions for the controllability of linear systems are derived. Consequently, sufficient conditions for nonlinear systems are established with the natural assumption that the linear system is controllable by using Schauder's fixed point theorem. To show the effectiveness of the theory, examples are provided.

\section{Acknowledgment}

The authors wish to thank the referees for many helpful suggestions.

\section{References}

Adams, J.L. and Hartley, T.T. (2008). Finite time controllability of fractional order systems, Journal of Computational and Nonlinear Dynamics 3(2): 021402-1-021402-5.

Al Akaidi, M. (2008). Fractal Speech Processing, Cambridge University Press, Cambridge.

Arena, P., Caponetta, R., Fortuna L. and Porto, D. (2008). Nonlinear Noninteger Order Circuits and Systems: An Introduction, World Scientific Series on Nonlinear Science, Vol. 38, World Scientific, Singapore.

Balachandran, K. and Dauer, J.P. (1987). Controllability of nonlinear systems via fixed point theorems, Journal of Optimization Theory and Applications 53(3): 345-352.

Balachandran, K. and Kiruthika, S. (2010). Existence of solutions of abstract fractional impulsive semilinear evolution equations, Electronic Journal of Qualitative Theory of Differential Equations 4: 1-12.

Balachandran, K. and Trujillo, J.J. (2010). The nonlocal Cauchy problem for nonlinear fractional integrodifferential equations in Banach spaces, Nonlinear Analysis: Theory, Methods and Applications 72(12): 4587-4593.

Balachandran, K., Kiruthika, S. and Trujillo, J.J. (2011). Existence results for fractional impulsive integrodifferential equations in Banach spaces, Communication in Nonlinear Science and Numerical Simulation 16(4): 1970-1977.

Balachandran, K., Park J.Y. and Trujillo, J.J. (2012). Controllability of nonlinear fractional dynamical systems, Nonlinear Analysis: Theory, Methods \& Applications 75(4): 19191926.

Benson, D.A., Wheatcraft, S.W. and Meerschaert, M.M. (2000). Application of a fractional advection-dispersion equation, Water Resources Research 36(6): 1403-1412.

Bettayeb, M. and Djennoune, S. (2008). New results on the controllability and observability of fractional dynamical systems, Journal of Vibrating and Control 14(9-10): 15311541.

Bonilla, B., Rivero, M. and Trujillo, J.J. (2007). On systems of linear fractional differential equations with constant coefficients, Applied Mathematics and Computation 187(1): 68-78.
Caputo, M. (1967 ). Linear model of dissipation whose $Q$ is almost frequency independent, Part II, Geophysical Journal of Royal Astronomical Society 13(5): 529-539.

Chen, Y.Q., Ahn, H.S. and Xue, D. (2006). Robust controllability of interval fractional order linear time invariant systems, Signal Processing 86(10): 2794-2802.

Chikrii, A.A. and Matichin, I.I. (2008). Presentation of solutions of linear systems with fractional derivatives in the sense of Riemann-Liouville, Caputo and Miller-Ross, Journal of Automation and Information Sciences 40(6): 1-11.

Chikrii, A. and Matichin, I.I. (2010). Game problems for fractional order systems, in D. Baleanu, Z.B. Guvenc and J.A.T. Machado (Eds.), New Trends in Nanotechnology and Fractional Calculus, Springer-Verlag, New York, NY, pp. 233-241.

Do, V.N. (1990). Controllability of semilinear systems, Journal of Optimization Theory and Applications 65(1): 41-52.

Guermah, S.A., Djennoune, S.A. and Bettayeb, M.A.(2008) Controllability and observability of linear discrete time fractional order systems, International Journal of Applied Mathematics and Computer Science 18(2): 213-222, DOI: 10.2478/v10006-008-0019-6.

Herrmann, R. (2011). Fractional Calculus: An Introduction for Physicists, World Scientific Publishing, Singapore.

Ichise, M., Nagayanagi, Y. and Kojima, T. (1971). Analog simulation of non integer order transfer functions for analysis of electrode processes, Journal of Electroanalytical Chemistry 33(2): 253-265.

Karthikeyan, S. and Balachandran, K. (2011). Constrained controllability of nonlinear stochastic impulsive systems, International Journal of Applied Mathematics and Computer Science 21(2): 307-316, DOI: 10.2478/v10006-011 -0023-0.

Kexue, L. and Jigen, P. (2011). Laplace transform and fractional differential equations, Applied Mathematics Letters 24(12): 2019-2023

Kilbas, A.A., Srivastava, H.M. and Trujillo, J.J. (2006). Theory and Applications of Fractional Differential Equations, Elsevier, New York, NY.

Klamka, J. (1993). Controllability of Dynamical Systems, Kluwer Academic, Dordrecht.

Klamka, J. (2008). Constrained controllability of semilinear systems with delayed controls, Bulletin of the Polish Academy of Sciences: Technical Sciences 56(4): 333-337.

Klamka, J. (2010). Controllability and minimum energy control problem of fractional discrete time systems, in $\mathrm{D}$. Baleanu, Z.B. Guvenc and J.A.T. Machado (Eds.), New Trends in Nanotechnology and Fractional Calculus, SpringerVerlag, New York, NY, pp. 503-509.

Liu, F., Anh, V.V., Turner, I. and Zhuang, P. (2003). Time fractional advection-dispersion equation, Journal of Applied Mathematics and Computing 13(1-2): 233-245.

Machado, J.T., Kiryakova, V. and Mainardi, F. (2011). Recent history of fractional calculus, Communications in Nonlinear Science and Numerical Simulations 16(3): 1140-1153. 
Machado, J.T. (1997). Analysis and design of fractional order digital control systems, Systems Analysis, Modelling and Simulation 27(2-3): 107-122.

Metzler, R. and Klafter, J. (2000). The random walk's guide to anomalous diffusion: A fractional dynamics approach, Physics Reports 339(1): 1-77.

Miller, K.S. and Ross, B. (1993). An Introduction to the Fractional Calculus and Fractional Differential Equations, Wiley, New York, NY.

Monje, C.A., Chen, Y.Q., Vinagre, B.M., Xue, D. and Feliu, V. (2010). Fractional-order Systems and Controls: Fundamentals and Applications, Springer, London.

Oldham, K.B., and Spanier, J. (1974). The Fractional Calculus, Academic Press, New York, NY.

Oustaloup, A. (1991). La Commade CRONE: Commande Robuste d'Ordre Non Entier, Hermès, Paris.

Podlubny, I. (1999a). Fractional Differential Equations, Academic Press, London.

Podlubny, I. (1999b). Fractional-order systems and $P I^{\lambda} D^{\mu}$ controllers, IEEE Transactions on Automatic Control 44(1): 208-214.

Renardy, M., Hrusa, W.J. and Nohel, J.A.(1987). Mathematical Problems in Viscoelasticity, Longman Scientific and Technical, New York, NY.

Samko, S.G., Kilbas, A.A. and Marichev, O.I. (1993). Fractional Integrals and Derivatives: Theory and Applications, Gordan and Breach, Amsterdam.

Shamardan, A.B. and Moubarak, M.R.A. (1999). Controllability and observability for fractional control systems, Journal of Fractional Calculus 15(1): 25-34.

Valerio, D. and Sa da Costa, J. (2004). Non integer order control of a flexible robot, Proceedings of the IFAC Workshop on Fractional Differentiation and its Applications, Bordeaux, France, pp. 520-525.

West, B.J., Bologna, M. and Grigolini, P. (2003). Physics of Fractal Operators, Springer-Verlag, Berlin.

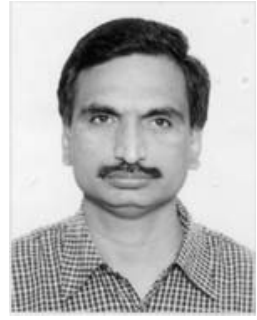

Krishnan Balachandran is a professor in the Department of Mathematics, Bharathiar University, Coimbatore, India. He received the M.Sc. degree in mathematics in 1978 from the University of Madras, Chennai, India. He obtained his M.Phil. and Ph.D. degrees in applied mathematics in 1980 and 1985, respectively, from the same university. In the years 1986-1988, he worked as a lecturer in mathematics at Madras University, P.G. Centre at Salem. In 1988, he joined Bharathiar University, Coimbatore, as a reader in mathematics and subsequently was promoted to a professor of mathematics in 1994. He received the Fulbright Award (1996), the Chandna Award (1999), and the Tamil Nadu Scientists Award (1999) for his research contributions. He has served as a visiting professor at Sophia University, Japan, Pusan National University, South Korea, and Yonsei University, South Korea. He has published more than 300 technical papers in learned journals. His major research areas include control theory, abstract integro-differential equations, stochastic differential equations, fractional differential equations, and partial differential equations. He is also a member of the editorial board of the journal Nonlinear Analysis: Hybrid Systems.

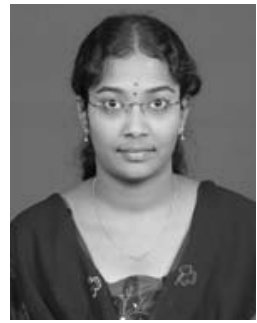

Jayakumar Kokila received the B.Sc. degree in mathematics from Bharathiar University, Coimbatore, India, in 2006. She obtained her M.Sc. and M.Phil. degrees in mathematics in 2008 and 2009, respectively, from the same university. Now, she is pursuing her Ph.D. study under the guidance of Prof. K. Balachandran at Bharathiar University. Her research interests focus on the analysis and control of fractional dynamical systems.

Received: 3 June 2011

Revised: 4 November 2011 